\title{
I disordini endocrini nella pratica clinica: epidemiologia e domanda di salute nella vita reale
}

\author{
Andrea Crafa $^{1} \cdot$ Aldo E. Calogero $^{1} \cdot$ Rosita A. Condorelli ${ }^{1} \cdot$ Rossella Cannarella $^{1} \cdot$ Sandro La Vignera $^{1}$
}

Accettato: 25 febbraio 2021 / Pubblicato online: 7 settembre 2021

(c) The Author(s) 2021

Sommario Le patologie del sistema endocrino hanno un notevole impatto dal punto di vista epidemiologico sulla salute delle popolazioni. Esse sono in grado di alterare la qualità della vita dei pazienti affetti e sono responsabili di disabilità a lungo termine; si collocano al quinto posto tra le cause di morte. In questa revisione della letteratura abbiamo valutato la prevalenza e l'incidenza delle principali malattie endocrine nel mondo e in Italia per evidenziarne il reale impatto nella pratica clinica dell'endocrinologo.

Parole chiave Prevalenza delle endocrinopatie - Incidenza delle endocrinopatie $\cdot$ Epidemiologia in endocrinologia

\section{Introduzione}

Dai dati dell'Osservatorio Europeo Delle Politiche e Dei Sistemi Sanitari redatti in forma di report emerge che in Italia le patologie cardiovascolari (40\% nelle donne e $35 \%$ negli uomini) seguite da quelle oncologiche (24\% nelle donne e $33 \%$ negli uomini) rappresentano le cause più frequentemente ricollegabili a eventi di successiva mortalità. Le malattie endocrine ( $5 \%$ nelle donne e $4 \%$ negli uomini) si collocano al quinto posto di questo elenco, dopo le cause respiratorie ( $6 \%$ nelle donne e $8 \%$ negli uomini) e quelle neurologiche (8\% nelle donne e 5\% negli uomini) [1]. Que-

Proposto da Antonio Aversa.

Informazioni Supplementari La versione online contiene materiale supplementare disponibile su

https://doi.org/10.1007/s40619-021-00961-x.

S. La Vignera

sandrolavignera@unict.it

1 Dipartimento di Medicina Clinica e Sperimentale, Università di Catania, Catania, Italia sto impatto sul rischio di mortalità è da attribuire agli importanti risvolti sistemici della maggior parte delle malattie endocrine (Tabella 1).

Sulla base di queste premesse, abbiamo ricercato i dati di incidenza e prevalenza dei principali disturbi di interesse endocrinologico per rilevare il loro effettivo impatto nella pratica clinica quotidiana. Nel dettaglio, i dati di prevalenza e incidenza delle singole patologie sono stati estratti, quando possibile, da registri nazionali e internazionali come quelli dell'Istituto Nazionale di Statistica (ISTAT), dell'Istituto Superiore di Sanità (ISS), dell'Osservatorio ARNO, dell'Organizzazione Mondiale della Sanità (OMS). Nel caso di impossibilità a reperire $\mathrm{i}$ dati da queste fonti, sono state utilizzate Linee Guida di società scientifiche internazionali o rassegne che sintetizzassero le evidenze di studi condotti in diverse aree geografiche del mondo e in Italia. Sono stati inclusi prevalentemente lavori condotti nell'ultimo ventennio (2000-2020), prediligendo tuttavia, nella selezione del dato di prevalenza, quelli condotti nell'ultimo quadriennio (2017-2020).

\section{Prevalenza e incidenza delle patologie ipofisarie}

La patologia ipofisaria a maggiore prevalenza è rappresentata dagli adenomi ipofisari. La diagnosi precoce e l'eventuale trattamento di queste masse risulta fondamentale dal punto di vista clinico, dal momento che possono produrre ormoni e determinare effetto massa (cefalea, alterazioni del campo visivo, vomito, ecc.). La prevalenza generale si attesta intorno a 1 caso ogni 865-2688 persone, anche se gli studi autoptici rilevano la presenza di piccoli adenomi ipofisari in circa il 10\% delle autopsie [2]. In $\sim 50 \%$ dei casi si tratta di microadenomi (dimensione $<10 \mathrm{~mm}$ ); i restanti sono macroadenomi. Da un punto di vista funzionale, 
Tabella 1 Manifestazioni sistemiche delle principali patologie endocrinologiche

\begin{tabular}{ll}
\hline Patologia & Manifestazioni sistemiche \\
\hline Ipotiroidismo & Bradicardia, ipertensione diastolica, anemia, aumento ponderale, ipercolesterolemia \\
Ipertiroidismo & Tachicardia, ipertensione sistolica, fibrillazione atriale, osteoporosi, calo ponderale \\
Ipoparatiroidismo & Aritmie cardiache (prolungamento QT), calcificazioni tissutali (gangli della base, nefrocalcinosi) \\
Iperparatiroidismo primario & $\begin{array}{l}\text { Osteoporosi, nefrolitiasi, insufficienza renale, pancreatite e manifestazioni non classiche (cardiovascolari, } \\
\text { gastrointestinali, neuromuscolari e articolari, neuropsicologiche e cognitive) }\end{array}$ \\
Morbo di Addison & $\begin{array}{l}\text { Ipotensione, ipoglicemia e squilibri elettrolitici (in particolare nelle forme associate anche a deficit dei } \\
\text { mineralcorticoidi) }\end{array}$ \\
Sindrome di Cushing & Ipertensione, diabete mellito, obesità, osteoporosi \\
Iperaldosteronismo & Ipertensione, squilibri elettrolitici con ipernatriemia e ipokaliemia che possono determinare aritmie fatali \\
Feocromocitoma & Ipertensione, ipotensione ortostatica, aritmie fatali, alterazione del metabolismo glucidico \\
Acromegalia & Ipertensione, iperglicemia, scompenso cardiaco, aumentato rischio tumorale, dislipidemia \\
Ipogonadismo maschile & Osteoporosi, alterazione del metabolismo glucidico, obesità, anemia \\
Diabete mellito & Complicanze micro e macrovascolari (retinopatia, nefropatia, neuropatia, incremento del rischio di eventi \\
& cardiovascolari)
\end{tabular}

nel 32-66\% dei casi si tratta di prolattinomi, nell' $8-16 \%$ di GHomi, nel 2-6\% ACTHomi e in circa 1'1\% di TSHomi [2]. Gli adenomi non funzionanti sono presenti nel 15-54\% dei casi, con prevalenza di 7-41,3/100.000 e incidenza annua di 0,65-2,34/100.000 abitanti [3].

L'altra condizione ipofisaria di interesse clinico è rappresentata dall'ipopituitarismo. Questo disturbo si caratterizza per il deficit parziale o totale di produzione degli ormoni ipofisari che si configurano in quadri di insufficienza surrenalica, ipotiroidismo, ipogonadismo, deficit di ormone della crescita e, in casi più rari, diabete insipido. I pazienti con ipopituitarismo, specialmente quelli con deficit ormonali multipli, rappresentano un'importante voce della spesa sanitaria e richiedono l'intervento di centri specializzati al fine di garantire la migliore qualità di vita per questi pazienti. La prevalenza del disturbo si attesta intorno ai 45 casi/100.000 persone con incidenza annua di 4 casi/100.000 abitanti [4].

\section{Prevalenza e incidenza delle patologie tiroidee}

Le disfunzioni tiroidee sono comuni e, in genere, facilmente trattabili; tuttavia, se non diagnosticate possono compromettere in maniera importante la salute dell'individuo in virtù del ruolo fondamentale che gli ormoni tiroidei svolgono nel regolare la frequenza cardiaca, la funzione digestiva, lo sviluppo fisico, lo sviluppo e il funzionamento cerebrale e il metabolismo cellulare in generale [5].

La patologia tiroidea con prevalenza più alta è rappresentata senz'altro dal nodulo tiroideo. Infatti, dal punto di vista ecografico, il riscontro di nodularità tiroidea si verifica nel 19-68\% della popolazione generale con maggiore prevalenza nelle donne e negli anziani. Tuttavia, solo il 5\% dei noduli nelle donne e 1' $1 \%$ dei noduli negli uomini è palpabile e, dunque, diagnosticabile al semplice esame obiet- tivo. Infine, solo nel $7 \%$ dei casi un nodulo tiroideo risulta poi essere maligno all'esame istologico, con una prevalenza del $90 \%$ di forme differenziate (carcinoma papillare o follicolare) [6]. L'incidenza di cancro tiroideo negli ultimi anni sembra essere aumentata, probabilmente ma non soltanto per una migliore capacità diagnostica conseguente l'utilizzo dell'esame ecografico. Negli USA, si registrano ad oggi circa 14,3/100.000 abitanti/anno [6], mentre in Italia l'incidenza varia da 7 a 37,5/100.000 abitanti/anno a seconda della regione [7].

Un'altra disfunzione tiroidea molto prevalente, che interessa circa l'1-2\% della popolazione generale, è l'ipotiroidismo. La principale causa di questa patologia nel mondo è rappresentata ancora oggi dalla carenza di iodio. Tuttavia, nelle regioni iodo-sufficienti l'ipotiroidismo primario può essere ascritto a cause congenite, oppure a cause acquisite come quella autoimmune o iatrogena (secondaria a intervento di tiroidectomia o radioiodioterapia). Molto più rare sono, invece, le forme secondarie, da deficit di produzione di tireotropina (TSH). L'incidenza di ipotiroidismo è aumentata dal 1995 ad oggi passando da 3,5 casi/1.000/anno nelle donne e 0,6 casi/1.000/anno negli uomini a circa 7 casi/1.000/anno, probabilmente per l'aumento del numero di pazienti sottoposti a tiroidectomia [8].

Infine, l'ipertiroidismo interessa circa lo 0,2-1,3\% della popolazione generale nei Paesi iodo-sufficienti, con un'incidenza annua di circa 51 casi/100.000 abitanti. La principale causa di ipertiroidismo è rappresentata dal morbo di Flajani-Graves-Basedow, seguito dal gozzo multinodulare tossico e dall'adenoma di Plummer. Più rare sono invece le forme conseguenti a tiroidite, ad adenoma ipofisario TSH secernente e le forme farmaco-indotte [5]. In Italia, un recente studio ha stimato una prevalenza di ipertiroidismo in 756/100.000 abitanti con una incidenza di 81 casi su 100.000 persone per anno [9]. 


\section{Prevalenza e incidenza delle patologie delle paratiroidi}

L'ipoparatiroidismo cronico è una condizione clinica piuttosto rara caratterizzata da carenza di produzione di ormone paratiroideo $(\mathrm{PTH})$ che richiede il trattamento con sali di calcio e Vitamina D per più di 6 mesi. Nella maggior parte dei casi è di natura iatrogena conseguente a danno non intenzionale delle paratiroidi durante intervento chirurgico. Rare sono invece le varianti autoimmuni o geneticamente determinate. Gli studi epidemiologici sull'ipoparatiroidismo cronico sono carenti. Negli Stati Uniti, è stata stimata una prevalenza di 25-37 casi/100.000 abitanti, mentre in Danimarca la prevalenza è di 25,4 casi/100.000 abitanti. In Italia, uno studio toscano ha stimato una prevalenza di $27 \mathrm{ca}-$ si/100.000 abitanti, in linea con quelli che sono i dati nel resto del mondo [10].

Notevolmente maggiore è, invece, la prevalenza dell'iperparatiroidismo primario con 3 casi 1.000 abitanti nella popolazione generale che aumenta a 21 casi/1.000 nella fascia di età compresa tra 55 e 75 anni [11].

\section{Prevalenza e incidenza delle patologie surrenaliche}

La patologia surrenalica a maggiore prevalenza è rappresentata dall'incidentaloma surrenalico. Per definizione, l'incidentaloma surrenalico è una massa surrenalica che viene diagnosticata casualmente in seguito a esame di diagnostica per immagini. Gli studi autoptici stimano una prevalenza di questa patologia in circa il $2 \%$ della popolazione con range compreso tra 1 e $8,7 \%$ che aumenta con l'età. Radiologicamente, la prevalenza si attesta al $3 \%$ con aumento al $10 \%$ nella popolazione anziana [12]. Nell' $85 \%$ dei casi si tratta di adenomi non funzionanti, nel $10 \%$ dei casi determinano sindrome di Cushing, nel 4,2\% si tratta di feocromocitomi e nell' $1,6 \%$ di aldosteronomi [13]. L' $8 \%$ delle masse surrenaliche si rivela, inoltre, essere un carcinoma surrenalico, con un'incidenza annua di questa condizione di 0,7-2 casi/1.000.000 abitanti [14].

Più rara è, invece, la malattia di Addison. Questo disturbo interessa circa 82-144/1.000.000 di abitanti per anno con un'incidenza annuale di 4,5 casi/1.000.000 [14]. In passato, tale disfunzione era strettamente collegata all'infezione tubercolare, mentre oggi la forma principale è quella autoimmune che, a sua volta, può essere spuria o associata ad altre condizioni autoimmuni nelle cosiddette sindrome poliendocrine autoimmunitarie (SPA). Più rare sono invece le forme post-infettive o le forme geneticamente determinate [15]. La malattia di Addison nella sua espressione più grave, la crisi surrenalica, può mettere seriamente in pericolo la vita del paziente. Si stima un'incidenza annuale di $6-8$ crisi/100 pazienti con un tasso di morte di $0,5 / 100$ pazienti [14]. In Italia, la prevalenza di malattia di Addison si attesta intorno ai 117 casi/1.000.000 di abitanti [15].

La condizione opposta, caratterizzata dall'iperproduzione endogena di cortisolo, è denominata Sindrome o Malattia di Cushing. La prevalenza di tale patologia si attesta a circa $10-15$ casi ogni 1.000 .000 di abitanti [16] con un un'incidenza variabile tra 0,7-2,4/1.000.000 di abitanti/anno [17]. Il Cushing può essere classificato in forme ACTHdipendenti (75-80\% dei casi) e forme ACTH-indipendenti (15-20\% dei casi). Le prime, a loro volta, sono correlate nel $75-80 \%$ dei casi alla presenza di adenomi ipofisari ACTH secernenti (Malattia di Cushing), nel 15-20\% dei casi a produzione ectopica di ACTH (microcarcinoma polmonare, tumori neuroendocrini, ecc.), mentre in $<1 \%$ dei casi abbiamo forme CRH-dipendenti. Invece, la Sindrome di Cushing ACTH-indipendente è causata nel $90 \%$ dei casi da tumori surrenalici che nell' $80 \%$ dei casi si rivelano essere adenomi. Esistono, poi, altre rare forme di Cushing surrenalico quali l'iperplasia surrenalica macronodulare, la malattia surrenalica nodulare pigmentata primaria (sporadica o come parte del complesso di Carney) e la sindrome di McCune-Albright [17].

Infine, nel contesto delle patologie surrenaliche riveste grande importanza l'iperplasia surrenalica congenita (CAH), al punto da essere stata inserita nello screening neonatale. Tale patologia è causata nel $90 \%$ dei casi da un deficit della $21 \alpha$-idrossilasi. A seconda del tipo di mutazione e dell'enzima coinvolto, lo spettro sintomatologico della patologia è estremamente vario, da quadri severi e potenzialmente letali con crisi da perdita di sali ( 8,8 casi/ 100 pazienti) a forme virilizzanti $(2,5$ casi/100 pazienti) $[14,18]$. La prevalenza delle forme classiche di CAH si attesta intorno a 1 caso/15.000 [18] con un'incidenza annuale di crisi surrenaliche dovute alla CAH di circa 5,8 casi/100 pazienti [14]. In Italia, si stima una prevalenza di $1 \mathrm{caso} / 13.000-22.000$ [14]. Esistono poi delle forme non classiche (NC-CAH), che vengono diagnosticate in età adulta, dovute a mutazioni del gene meno espressive. La prevalenza di queste forme è superiore rispetto alla variante classica, attestandosi nella popolazione caucasica a 1 caso/1.000 [18].

\section{Prevalenza e incidenza delle patologie gonadali, dell'infertilità e delle disfunzioni sessuali}

L'ipogonadismo maschile è un disturbo caratterizzato dalla ridotta funzione testicolare con conseguente deficit di produzione di androgeni, inibina $\mathrm{B}$, ormone anti-Mülleriano $(\mathrm{AMH})$ e insufficienza spermatogenetica. A seconda dell'età di insorgenza del deficit della funzione testicolare la manifestazione della patologia è differente. Prima della pubertà, 
l'alterato funzionamento testicolare può essere diagnosticato sulla base dei bassi livelli di AMH e inibina B. Al momento della pubertà, l'ipogonadismo si manifesta come ritardo puberale. Solo raramente l'ipogonadismo può rendersi clinicamente manifesto già alla nascita con anomalie genitali (1 caso/4.500 nati).

La principale causa di ipogonadismo primario geneticamente determinato è rappresentata dalla sindrome di Klinefelter con una prevalenza di 1/500-1.000 nati. Tra le forme acquisite, abbiamo invece quelle secondarie a chemioterapia e radioterapia, e le forme funzionali associate a malattie croniche che presentano un'incidenza annua di 1/10.000-100.000. La forma acquisita a maggiore prevalenza, strettamente connessa all'età, è rappresentata dall'ipogonadismo a insorgenza tardiva ( $\mathrm{LOH})$. Tale condizione interessa circa il 2,1\% della popolazione maschile di età compresa tra i 40 e i 79 anni ed è collegata sia al fisiologico declino di testosterone che si verifica nel corso della vita, sia all'aumento di peso e allo sviluppo di patologie croniche che spesso contraddistinguono l'avanzare dell'età [19]. Altra forma acquisita di ipogonadismo è quello secondario a orchiectomia per tumore testicolare. Questo rappresenta l'1-2\% di tutte le neoplasie maligne negli uomini ed è la più comune neoplasia maligna tra i giovani uomini. L'incidenza varia da 0,5 a 9,2/100.000 persone/anno [20]; in Italia, l'incidenza è di 7 casi ogni 100.000/anno [21].

La disfunzione ovarica dell'età fertile a maggiore prevalenza è rappresentata dalla sindrome dell'ovaio micropolicistico (PCOS). Tale disturbo si caratterizza per il notevole impatto clinico in quanto non solo causa irregolarità mestruali, infertilità e segni clinici o biochimici di iperandrogenismo, ma si associa anche ad alterazioni del metabolismo glucidico (insulino-resistenza e alterata tolleranza al glucosio) e lipidico che, a loro volta, sono importanti fattori di rischio cardiovascolari [22]. Il disturbo interessa circa il 7\% delle donne in età fertile [23], anche se si stima che il $75 \%$ delle donne con PCOS non venga diagnosticata durante la visita con il proprio medico, probabilmente a causa dell'estrema variabilità della presentazione clinica [22]. Nelle donne obese, la prevalenza di PCOS è maggiore, raggiungendo anche il $15-30 \%$ [22].

La riduzione della funzione ovarica al di sotto dei 40 anni prende il nome di insufficienza ovarica prematura (POF) e interessa circa l' $1 \%$ delle donne. Le forme spontanee riconoscono generalmente un'eziologia autoimmunitaria o genetica. Esistono poi le forme acquisite conseguenti a terapia radiante o con chemioterapici e le forme iatrogene conseguenti a ovariectomia per tumore ovarico [24]. Quest'ultimo presenta in Italia un'incidenza annuale di 15,2 casi ogni 100.000 donne [21].

Altra condizione di grande interesse endocrinologico e, in parte, connessa con i disturbi precedentemente elencati è rappresentata dall'infertilità. Si stima che il 15\% delle coppie nel mondo sia affetto da infertilità per un totale di circa
48,5 milioni di coppie. Nel 30\% dei casi l'infertilità è da attribuire a fattore maschile, nel $50 \%$ dei casi a fattore femminile e nel $20 \%$ dei casi sono coinvolti entrambi i partner [25]. In totale, dunque, la percentuale di uomini affetti da infertilità è variabile dal 2,5 al $12 \%$ con più di 30.000 .000 di uomini infertili in tutto il mondo [25]. Sul versante femminile, invece, si stima che circa il $2 \%$ delle donne di età compresa tra 20 e 44 anni sia affetta da infertilità primaria e che un altro $10,5 \%$ di donne in età fertile sperimenti una condizione di infertilità secondaria [26].

Per quanto riguarda l'ambito sessuologico, grande impatto dal punto di vista epidemiologico hanno le disfunzioni sessuali. Si stima che circa il $40 \%$ delle donne in tutto il mondo presenti disfunzioni sessuali, ma solo nel 12-25\% dei casi tali disturbi creano distress personale [27]. La disfunzione a maggiore prevalenza è rappresentata dal desiderio sessuale ipoattivo che, negli USA, interessa il 39\% delle donne [28], mentre in Europa è riportata una prevalenza del 29\% [29]. Gli altri disturbi con maggiore prevalenza sono la bassa eccitazione (26\%) e i disturbi dell'orgasmo $(21 \%)$ [28]. Questi disturbi rientrano in parte tra quelli presenti nel periodo peri e post-menopausale. Ogni anno il numero di donne in menopausa aumenta di circa 47.000 unità e si stima che entro il 2030 saranno circa 1,2 miliardi le donne in menopausa nel mondo [30]. L'80\% di queste donne presenta disturbi legati alle variazioni ormonali connesse alla menopausa (sintomi genito-urinari, sintomi vasomotori, sintomi cognitivi), ma solo nel $25 \%$ dei casi viene richiesta assistenza medica [31].

$\mathrm{Nel}$ sesso maschile la disfunzione sessuale a prevalenza più elevata è l'eiaculazione precoce. Questa interessa circa il $30 \%$ della popolazione maschile in tutto il mondo [32]. In Italia, la prevalenza si attesta intorno al $20 \%$ della popolazione maschile di età superiore a 18 anni; tuttavia, solo il 9\% dei maschi si rivolge a un medico [33]. L'altra disfunzione sessuale con grande impatto psico-fisico è rappresentata dalla disfunzione erettile, con un'incidenza di 12,4/1.000 anno negli uomini di età compresa tra 40 e 49 anni, 29,8/1.000 negli uomini di età compresa tra 50 e 59 anni, 46,4/1.000 negli uomini di età compresa tra 60 e 69 anni [34]. La prevalenza è di circa il $20 \%$ nella fascia di età al di sotto dei 30 anni, $25 \%$ tra 30 e 39 anni, $40 \%$ tra 40 e 49 anni, $60 \%$ tra 50 e 59 anni, $80 \%$ tra 60 e 69 anni e $90 \%$ nei maschi di età $>70$ anni [35]. In Italia, la prevalenza media del disturbo si attesta al 12,8\% [36]. Tuttavia, anche in questo caso il $70 \%$ dei pazienti non riceve consulenza medica [37].

Sempre nell'ambito della sessualità rientra il disturbo di identità di genere o disforia di genere. Secondo il DSM5, tale disturbo interessa lo $0,005-0,014 \%$ dei maschi biologici e $0,002-0,003 \%$ delle femmine biologiche [38].

Per concludere con i disturbi della sfera ormonale, una menzione va fatta ai disordini dello sviluppo nei quali rientrano la pubertà precoce e il ritardo puberale. La pubertà 
precoce è una condizione caratterizzata dalla comparsa di caratteri sessuali secondari prima degli 8 anni nelle femmine e prima dei 9 anni nei maschi. Interessa circa 1/5.000 bambini con rapporto femmine/maschi di 10:1 [39]. La pubertà ritardata è un disturbo che determina importante stress psicologico sia per i pazienti affetti che per i loro genitori. Si caratterizza nelle donne per la mancanza di sviluppo mammario all'età di 13 anni, o per una differenza di più di 4 anni tra il telarca e il menarca. Nei ragazzi, si caratterizza per la mancanza di aumento del volume testicolare dopo i 14 anni o per una differenza di più di 4 anni tra l'inizio dello sviluppo testicolare e il completamento della pubertà. Interessa circa il 2\% degli adolescenti [40]. Nel 60\% dei casi si tratta di un ritardo costituzionale di crescita e pubertà, nel $10 \%$ è la conseguenza di ipogonadismo centrale, nel $7 \%$ è dovuto a ipogonadismo primario e, infine, nel $20 \%$ dei casi si tratta di forme di ipogonadismo funzionale (ipogonadismo dismetabolico, associato a patologie sistemiche croniche, ecc.) [19].

\section{Prevalenza e incidenza delle patologie metaboliche}

L'alterazione metabolica a maggiore prevalenza è rappresentata dalla dislipidemia e, in particolare, dall'ipercolesterolemia che duplica il rischio di eventi cardiovascolari [41]. Per questo motivo, la sua adeguata gestione è di fondamentale importanza. Negli Stati Uniti, il 53\% circa degli uomini adulti ha elevati livelli di LDL [41]. In Italia, in base ai dati del Progetto Cuore dell'ISS, il 21\% degli uomini e il $23 \%$ delle donne è ipercolesterolemico (popolazione di età compresa tra 34 e 74 anni); tuttavia, solo il $13 \%$ degli uomini e il $9 \%$ delle donne dichiara di essere trattato in maniera adeguata, mentre 1' $81 \%$ degli uomini e l' $85 \%$ delle donne dichiara di non aver ricevuto mai alcun trattamento [42]. Tra le forme genetiche, quelle a maggiore prevalenza sono l'ipercolesterolemia familiare (variante eterozigote), 1 caso ogni 200-250 persone, e l'iperlipidemia familiare combinata, 1 caso ogni 100-200 [43].

L'altra condizione metabolica con ampio risvolto sanitario, sociale ed economico è il diabete mellito (DM). Il DM colpisce più 425 milioni di persone nel mondo (prevalenza dell' $8,5 \%$ ), di cui solo il 5-10\% è attribuibile al DM di tipo I [44]. Secondo i dati del Rapporto dell'Osservatorio ARNO Diabete del 2019, tra il 6,2 e il 7,2\% degli italiani è diabetico, con circa 4 milioni di soggetti affetti. Tuttavia, un altro $1,5 \%$ dei casi sembrerebbe essere non diagnosticato, con circa 1 milione di italiani affetti che non sanno di avere la patologia o che non la trattano. Il 67\% dei casi di diabete interessa persone di età superiore ai 65 anni, l'1\% di età inferiore ai 20 anni e ben il 32\% in età lavorativa (20-64 anni). Questi dati evidenziano il notevole risvolto socio-economico correlato a tale patologia [45].
La principale condizione, che in parte si associa alle alterazioni metaboliche sopra descritte, è rappresentata dall'obesità. La prevalenza e l'incidenza di questo fenomeno è in crescita a causa di fattori evoluzionistici, biologici, psicologici, sociologici, economici e istituzionali [46]. Secondo l'OMS, nel 2016 le persone affette da obesità erano più di 1,9 miliardi [47]. In Italia, sono circa 25 milioni le persone in sovrappeso e obese, con una prevalenza di obesità del 10,8\% della popolazione [46]. Nel 2017, il sovrappeso e l'obesità sono stati responsabili di 148 milioni di anni vissuti con disabilità e di 4,72 milioni di decessi raggiungendo, così, il quarto posto fra le cause di morte, precedute soltanto da ipertensione, fumo e iperglicemia [46].

\section{Prevalenza e incidenza dei tumori neuroendocrini}

Una breve menzione, per quanto siano condizioni rare, va fatta ai tumori neuroendocrini (NET). Presentano una prevalenza di 2,2/1.000.000 di abitanti e un'incidenza di 1 caso/100.000 per anno. Nel $17 \%$ dei casi si tratta di insulinomi, nel $15 \%$ di gastrinomi. Più raramente possono essere VIPomi (2\%), glucagonomi (1\%), carcinoidi (serotonina $1 \%$ ) e somatostatinomi (1\%). Eccezionalmente possono produrre altri ormoni come $\mathrm{ACTH}$, calcitonina, peptide correlato al paratormone o altro ancora [48].

I tumori cromaffini, feocromocitomi e paragangliomi, hanno un'incidenza approssimativa di $2-8$ casi per milione di individui all' anno e sono responsabili di ipertensione in 1 caso su mille pazienti ipertesi.

\section{Prevalenza di osteoporosi}

L'osteoporosi è una condizione associata a elevata morbilità e mortalità. Ogni anno, l'osteoporosi causa più di 8,9 milioni di fratture, pari a una frattura ogni 3 secondi. Nel dettaglio, $1 / 3$ delle donne di età $>50$ anni e $1 / 5$ degli uomini di età $>50$ anni sviluppa fratture da osteoporosi [49]. Per comprende l'importanza di un'adeguata prevenzione e trattamento di questa condizione, basti pensare che in Europa la disabilità dovuta a osteoporosi è superiore a quella causata dai tumori (ad eccezione del cancro ai polmoni) ed è paragonabile o superiore a quella causata da malattie croniche come l'artrite reumatoide, l'asma e le malattie cardiache correlate all'ipertensione [49]. Infatti, le fratture da fragilità si posizionano al quarto posto tra le cause di morbilità cronica dopo malattie cardiache ischemiche, demenza e cancro ai polmoni, ma prima della broncopneumopatia cronica ostruttiva e dell'ictus ischemico. La frattura d'anca si associa a un rischio di mortalità del $20 \%$ nei primi 12 mesi dall'evento, mentre quella vertebrale a un incremento di circa 8 volte della mortalità età-aggiustata [50]. In Italia, 3,2 milioni di 


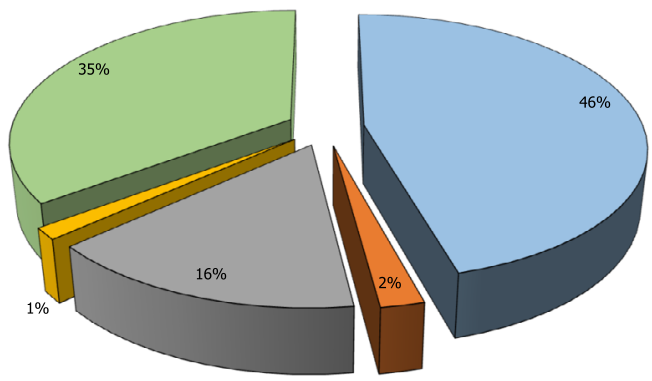

口Diabete gestazionale

口Ipertiroidismo

口Ipotiroidismo severo

口Tireopatia nodulare

Fig. 1 Prevalenza delle principali endocrinopatie della gravidanza. I dati sono stati calcolati applicando il dato di prevalenza al numero di gravidanze nel 2018 in Italia calcolato come somma del numero di parti, numero di aborti spontanei e numero di interruzioni volontarie di gravidanza [51, 52]

donne e 0,8 milioni di uomini sono affetti da osteoporosi, con una prevalenza del $23,1 \%$ delle donne $>50$ anni e del $7 \%$ degli uomini $>50$ anni [50].

\section{Prevalenza delle endocrinopatie della gravidanza}

Le endocrinopatie più frequenti della gravidanza sono quelle tiroidee e il diabete gestazionale (Fig. 1). La diagnosi di queste patologie in un periodo così delicato per la vita della donna si rende fondamentale, visti gli importanti risvolti materno-fetali che la mancata diagnosi di queste patologie può determinare.

La patologia endocrina gestazionale a maggiore prevalenza è rappresentata dal diabete gestazionale. Questo interessa circa $117 \%$ delle gravidanze e una sua non adeguata gestione si associa a importanti complicanze materno-fetali come ipertensione gestazionale, distacco di placenta, ritardo di crescita intrauterina con morte intrauterina e malformazioni congenite [53].

La tireopatia a maggiore prevalenza è rappresentata dalla patologia nodulare, in linea con la popolazione generale. Infatti, il riscontro di nodularità della tiroide si verifica nel 5\% circa delle gravidanze; tuttavia, data la bassa percentuale di noduli tiroidei maligni, quasi mai il loro riscontro durante la gestazione richiede interventi chirurgici urgenti o eventuale interruzione di gravidanza [54].

Più rare, ma clinicamente più gravi, sono invece le disfunzioni tiroidee. L'ipertiroidismo gestazionale si verifica in 1-2 gravidanze/1.000 con una prevalenza dello $0,2 \%$ circa. Le principali cause di ipertiroidismo gravidico sono sovrapponibili a quelle della popolazione generale, ad eccezione delle rare forme causate da eccesso di gonadotropina corionica umana. Nelle gestanti, l'ipertiroidismo può favorire l'ipertensione gestazionale, il distacco di placenta e l'aborto, mentre nel neonato può causare basso peso
Tabella 2 Elenco dei principali disturbi di interesse endocrinologico in ordine decrescente di frequenza

\begin{tabular}{ll}
\hline & Patologia \\
\hline 1 & Nodulo tiroideo rilevabile ecograficamente \\
2 & Obesità \\
3 & Dislipidemia \\
4 & Diabete mellito 2 \\
5 & Osteoporosi femminile \\
6 & Disfunzioni sessuali trattate \\
7 & Nodulo tiroideo maligno \\
8 & Disturbi della menopausa \\
9 & Infertilità femminile \\
10 & Ipotiroidismo \\
11 & Osteoporosi maschile \\
12 & PCOS \\
13 & Infertilità maschile \\
14 & Ipertiroidismo \\
15 & Ipogonadismo a insorgenza tardiva (LOH) \\
16 & Diabete mellito 1 \\
17 & Iperparatiroidismo \\
18 & Insufficienza ovarica precoce \\
19 & Pubertà ritardata \\
20 & Adenoma ipofisario \\
21 & Ipopituitarismo \\
22 & Ipoparatiroidismo \\
23 & Malattia di Addison \\
24 & Iperplasia surrenalica congenita \\
25 & Disforia di genere \\
\hline & Pubertà precoce \\
\hline & Tumori neuroendocrini \\
\hline & \\
\hline & \\
\hline &
\end{tabular}

alla nascita, prematurità, ipertiroidismo neonatale e morte intrauterina [54].

Una prevalenza più alta presenta, invece, l'ipotiroidismo che nella sua variante subclinica interessa il $2,2 \%$ delle donne in gravidanza, mentre nella forma di ipotiroidismo franco ha una frequenza nello $0,3 \%$ nelle donne gravide. Anche questa condizione favorisce l'ipertensione gestazionale, distacco di placenta, basso peso alla nascita, emorragia post-partum, malformazioni congenite e morte intrauterina [54].

La prevalenza delle altre patologie endocrinologiche in gravidanza è estremamente bassa, dal momento che molte disendocrinie possono alterare il normale processo ovulatorio. Ad esempio, le patologie ipofisarie sono piuttosto rare in gravidanza, dal momento che sia le iperfunzioni (prolattinomi, acromegalia, malattia di Cushing, ecc.), sia le ipofunzioni ipofisarie possono compromettere la fertilità. Rarissimi sono i casi descritti in letteratura di donne acromegaliche in gravidanza, dal momento che l'eccesso di ormone della crescita determina anovularietà. Il primo caso di gravidanza 
口Dislipidemia *

口Obesità

$\square \mathrm{DM} 2$

口Osteoporosi femminile

口Disfunzioni sessuali trattate **

$\square$ Nodulo tiroideo maligno

口Disturbi della menopausa severi ${ }^{* * *}$

口Incidentaloma surrene

口Infertilità femminile

口Ipotiroidismo

口Osteoporosi maschile

$\square \mathrm{PCOS}$

口Infertilità maschile

口Ipertiroidismo

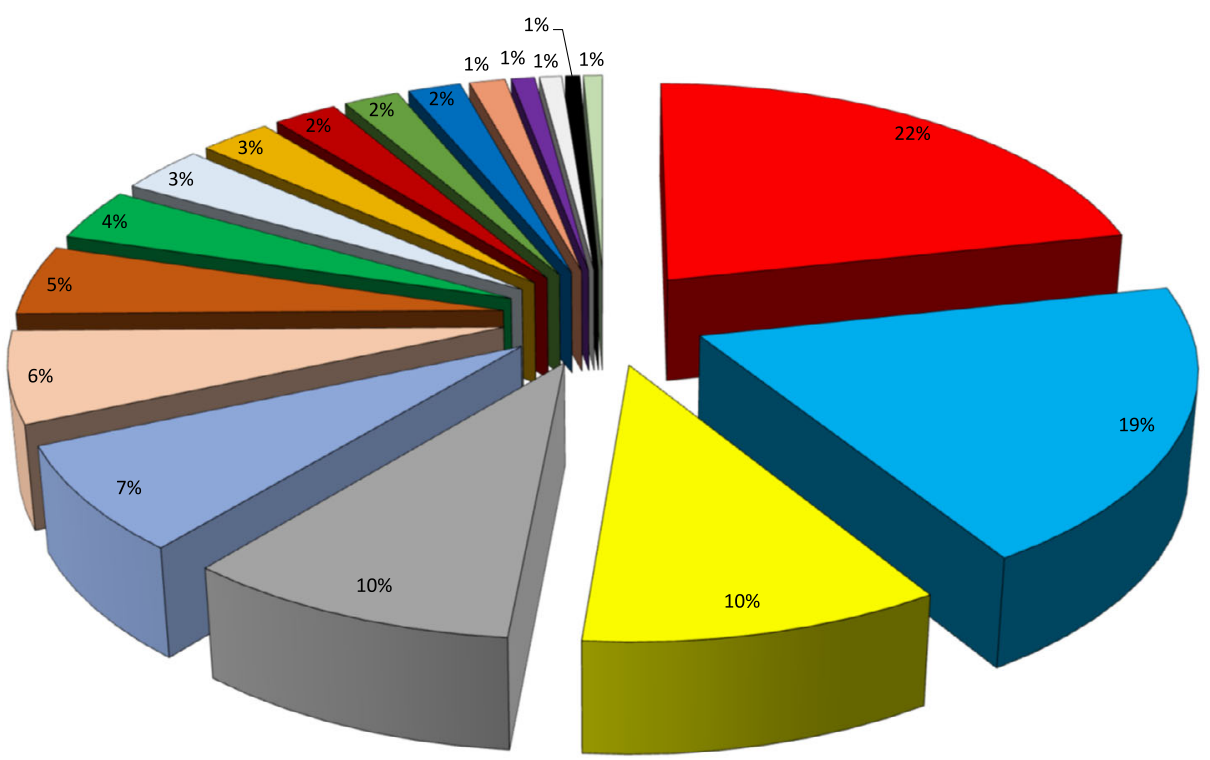

口LOH

$\square \mathrm{DM} 1$

- Iperparatiroidismo

口Altro (POF, Pubertà ritardata, Adenomi ipofisari, Ipopituitarismo, Ipoparatiroidismo, Addison, CAH, Disforia di genere, Pubertà precoce, Tumori neuroendocrini)

Fig. 2 Prevalenza dei principali disturbi di interesse endocrinologico nella popolazione italiana. *, nel calcolo è stata considerata la prevalenza di ipercolesterolemia (condizione più frequente); **, la prevalenza totale delle disfunzioni sessuali è maggiore di quella riportata nel grafico e supera nel complesso quella di altre condizioni come dislipi-

decorsa normalmente in paziente acromegalica fu descritto nel 1954. Anche l'ipopituitarismo è estremamente raro e i pochi casi descritti in letteratura si riferiscono a donne che si sono sottoposte a procedure di procreazione medicalmente assistita [55].

Per quanto riguarda le patologie delle paratiroidi, la prevalenza di iperparatiroidismo primario in gravidanza si attesta intorno allo $0,15 \%$. L'ipoparatiroidismo è anche più raro, manifestandosi principalmente come conseguenza di interventi di tiroidectomia. A testimonianza della rarità dell'evento, il primo caso di ipoparatiroidismo in gravidanza fu descritto nel 1942 [55].

Per quanto riguarda invece le patologie surrenaliche, ad oggi si contano circa 200 casi di sindrome di Cushing in gravidanza (sia ACTH-dipendente che ACTH-indipendente). La rarità di questa patologia nel periodo gestazionale deriva dal fatto che l'ipercortisolismo e l'iperandrogenismo presenti nella sindrome di Cushing alterano la fertilità. Anche la Malattia di Addison è piuttosto rara, con una prevalenza di circa 1 caso ogni 30.000 gravidanze. Ancora più raro è il feocromocitoma, con prevalenza dello $0,007 \%$ delle gestanti [55]. demia e obesità. Tuttavia, solo il 7\% di uomini e donne con disfunzioni sessuali viene trattato o comunque si rivolge a un medico per il trattamento del disturbo; ***, la menopausa interessa circa 10.000 .000 di donne. Di queste 1'80\% riferisce disturbi correlati alla condizione, ma solo il $25 \%$ richiede assistenza medica

\section{Conclusioni}

I dati di prevalenza sopra descritti applicati alla popolazione generale italiana e, per alcune patologie, in riferimento a specifiche popolazioni (bambini $<9$ anni e bambine $<8$ anni nella pubertà precoce, adolescenti per la pubertà ritardata, donne in menopausa per i disturbi menopausali, donne in età fertile per infertilità femminile e sindrome dell'ovaio policistico, donne di età compresa tra 15 e 40 anni per la POF, donne e uomini $>50$ anni per l'osteoporosi) elencati in Tabella 2 , permettono di valutare l'impatto in termine numerico percentuale dei vari disturbi di interesse endocrinologico (Fig. 2). In particolare, in base a questa elaborazione grafica dei dati, non stupisce che le patologie metaboliche (obesità, dislipidemia e DM2), l'osteoporosi e le tireopatie abbiano sicuramente l'impatto più elevato nella pratica clinica $\mathrm{e}$ come fondamentale sia, dunque, il ruolo dell'endocrinologo nell'adeguata gestione di questi pazienti. Tuttavia, in questa "classifica" anche i disturbi della sfera gonadale e sessuale rappresentano un'entità di notevole significato clinico, rendendo evidente l'importanza dell'endocrinologia andrologica e ginecologica per l'identificazione, gestione e tratta- 
mento specifico di questi pazienti, al fine di migliorarne la qualità di vita e prevenirne la morbilità futura.

Funding Note Open access funding provided by Università degli Studi di Catania within the CRUI-CARE Agreement.

Conflitto di interesse Gli autori Andrea Crafa, Aldo E. Calogero, Rosita A. Condorelli, Rossella Cannarella e Sandro La Vignera dichiarano di non avere conflitti di interesse.

Consenso informato Lo studio presentato in questo articolo non ha richiesto sperimentazione umana.

Studi sugli animali Gli autori di questo articolo non hanno eseguito studi sugli animali.

Nota della casa editrice Springer Nature rimane neutrale in riguardo alle rivendicazioni giurisdizionali nelle mappe pubblicate e nelle affiliazioni istituzionali.

Open Access This article is licensed under a Creative Commons Attribution 4.0 International License, which permits use, sharing, adaptation, distribution and reproduction in any medium or format, as long as you give appropriate credit to the original author(s) and the source, provide a link to the Creative Commons licence, and indicate if changes were made. The images or other third party material in this article are included in the article's Creative Commons licence, unless indicated otherwise in a credit line to the material. If material is not included in the article's Creative Commons licence and your intended use is not permitted by statutory regulation or exceeds the permitted use, you will need to obtain permission directly from the copyright holder. To view a copy of this licence, visit http://creativecommons.org/licenses/by/4.0/.

\section{Bibliografia}

1. Osservatorio Europeo Delle Politiche e Dei Sistemi Sanitari (2017) 9789264283428-en.pdf (oecd-ilibrary.org). Accessed on 7 February 2021

2. Molitch ME (2017) Diagnosis and treatment of pituitary adenomas: a review. JAMA 317(5):516-524

3. Ntali G, Wass JA (2018) Epidemiology, clinical presentation and diagnosis of non-functioning pituitary adenomas. Pituitary 21(2):111-118

4. Fleseriu M, Hashim IA, Karavitaki N et al (2016) Hormonal replacement in hypopituitarism in adults: an Endocrine Society clinical practice guideline. J Clin Endocrinol Metab 101(11):3888-3921

5. Taylor PN, Albrecht D, Scholz A et al (2018) Global epidemiology of hyperthyroidism and hypothyroidism. Nat Rev Endocrinol 14(5):301-316

6. Haugen BR, Alexander EK, Bible KC et al (2016) 2015 American Thyroid Association management guidelines for adult patients with thyroid nodules and differentiated thyroid cancer: the American Thyroid Association Guidelines Task Force on Thyroid Nodules and Differentiated Thyroid Cancer. Thyroid 26(1):1-133

7. Lise M, Franceschi S, Buzzoni C et al (for the AIRTUM Working Group) (2012) Changes in the incidence of thyroid cancer between 1991 and 2005 in Italy: a geographical analysis. Thyroid 22(1):27-34

8. Giorda CB, Carnà P, Romeo F et al (2017) Prevalence, incidence and associated comorbidities of treated hypothyroidism: an update from a European population. Eur J Endocrinol 176(5):533-542

9. Caputo M, Pecere A, Sarro A et al (2020) Incidence and prevalence of hyperthyroidism: a population-based study in the Piedmont Region. Italy Endocr 69(1):107-112
10. Cianferotti L, Parri S, Gronchi G et al (2018) Prevalence of chronic hypoparathyroidism in a Mediterranean region as estimated by the analysis of anonymous healthcare database. Calcif Tissue Int 103(2):144-150

11. Adami S, Marcocci C, Gatti D (2002) Epidemiology of primary hyperparathyroidism in Europe. J Bone Miner Res 17(Suppl 2):N18-23

12. Fassnacht M, Arlt W, Bancos I et al (2016) Management of adrenal incidentalomas: European Society of Endocrinology Clinical Practice Guideline in collaboration with the European Network for the Study of Adrenal Tumors. Eur J Endocrinol 175(2):G1-G34

13. Mantero F, Terzolo M, Arnaldi G et al (2000) A survey on adrenal incidentaloma in Italy. Study Group on Adrenal Tumors of the Italian Society of Endocrinology. J Clin Endocrinol Metab 85(2):637-644

14. Reincke M, Beuschlein F, Bornstein S et al (2019) The adrenal gland: central relay in health and disease. Exp Clin Endocrinol Diabetes 127(2-03):81-83

15. Betterle C, Presotto F, Furmaniak J (2019) Epidemiology, pathogenesis, and diagnosis of Addison's disease in adults. J Endocrinol Invest 42(12): 1407-1433

16. American Association of Neurological Surgeons. Cushing's Syndrome/Disease. https://www.aans.org/Patients/NeurosurgicalConditions-and-Treatments/Cushings-Disease. Accessed on 7 February 2021

17. Sharma ST, Nieman LK, Feelders RA (2015) Cushing's syndrome: epidemiology and developments in disease management. Clin Epidemiol 7:281-293

18. Gialluisi A, Menabò S, Baldazzi L et al (2018) A genetic epidemiology study of congenital adrenal hyperplasia in Italy. Clin Genet 93(2):223-227

19. Salonia A, Rastrelli G, Hackett $G$ et al (2019) Paediatric and adultonset male hypogonadism. Nat Rev Dis Primers 5(1):38

20. Manecksha RP, Fitzpatrick JM (2009) Epidemiology of testicular cancer. BJU Int 104(9 Pt B):1329-1333

21. I numeri del cancro in Italia. https://aiom.it/wp-content/uploads/ 2020/10/2020_Numeri_Cancro-operatori-web.pdf. Accessed on 7 February 2021

22. Wolf WM, Wattick RA, Kinkade ON, Olfert MD (2018) Geographical prevalence of polycystic ovary syndrome as determined by region and race/ethnicity. Int J Environ Res Public Health $15(11): 2589$

23. ACOG (2009) Practice Bulletin No. 108: polycystic ovary syndrome. Obstet Gynecol 114(4):936

24. Haller-Kikkatalo K, Uibo R, Kurg A, Salumets A (2015) The prevalence and phenotypic characteristics of spontaneous premature ovarian failure: a general population registry-based study. Hum Reprod 30(5):1229-1238

25. Agarwal A, Mulgund A, Hamada A, Chyatte MR (2015) A unique view on male infertility around the globe. Reprod Biol Endocrinol 13:37

26. Mascarenhas MN, Flaxman SR, Boerma T et al (2019) National, regional, and global trends in infertility prevalence since 1990: a systematic analysis of 277 health surveys. PLoS Med 9(12):e1001356

27. Palacios S, Castaño R, Grazziotin A (2009) Epidemiology of female sexual dysfunction. Maturitas 63(2):119-123

28. Shifren JL, Monz BU, Russo PA et al (2008) Sexual problems and distress in United States women: prevalence and correlates. Obstet Gynecol 112:970-978

29. Dennerstein L, Koochaki P, Barton I, Graziottin A (2006) Hypoactive sexual desire disorder in menopausal women: a survey of Western European women. J Sex Med 3:212-222

30. Hill K (1996) The demography of menopause. Maturitas 23(2):113-127

31. Pinkerton JV (2020) Hormone therapy for postmenopausal women. N Engl J Med 382(5):446-455 
32. Montorsi F (2005) Prevalence of premature ejaculation: a global and regional perspective. J Sex Med 2(Suppl 2):96-102

33. Porst H, Montorsi F, Rosen RC et al (2007) The premature ejaculation prevalence and attitudes (PEPA) survey: prevalence, comorbidities and professional help-seeking. Eur Urol $51: 816-824$

34. McKinlay JB (2000) The worldwide prevalence and epidemiology of erectile dysfunction. Int J Impot Res 12(Suppl 4):S6-S11

35. Allen MS, Walter EE (2019) Erectile dysfunction: an umbrella review of meta-analyses of risk-factors, treatment, and prevalence outcomes. J Sex Med 16(4):531-541

36. Parazzini F, Menchini Fabris F, Bortolotti A et al (2000) Frequency and determinants of erectile dysfunction in Italy. Eur Urol 37(1):43-49

37. Kubin M, Wagner G, Fugl-Meyer AR (2003) Epidemiology of erectile dysfunction. Int J Impot Res 15(1):63-71

38. Biondi M (ed) (2014) DSM-5. Manuale diagnostico e statistico dei disturbi mentali, Raffaello Cortina, Milan

39. Cesario SK, Hughes LA (2007) Precocious puberty: a comprehensive review of literature. J Obstet Gynecol Neonatal Nurs 36(3):263-274

40. Dye AM, Nelson GB, Diaz-Thomas A (2018) Delayed puberty. Pediatr Ann 47(1):e16-e22

41. Karr S (2017) Epidemiology and management of hyperlipidemia. Am J Manag Care 23(9 Suppl):S139-S148

42. Istituto superiore di sanità: Progetto cuore: Epidemiologia e prevenzione delle malattie cerebro e cardiovascolari. Colesterolo e ipercolesterolemia aspetti epidemiologici (iss.it). Accessed on 7 February 2021

43. Mach F, Baigent C, Catapano AL et al (2020) 2019 ESC/EAS Guidelines for the management of dyslipidaemias: lipid modification to reduce cardiovascular risk. ESC Scientific Document Group. Eur Heart J 41(1):111-188

44. Glovaci D, Fan W, Wong ND (2019) Epidemiology of diabetes mellitus and cardiovascular disease. Curr Cardiol Rep 21(4):21
45. Rapporto osservatorio ARNO diabete 2019. https://www.siditalia. it/clinica/linee-guida-societari/send/80-linee-guida-documentisocietari/5025-rapporto-arno-diabete-2019. Accessed on 7 February 2021

46. IBDO Foundation, Istat " 1 ${ }^{\circ}$ Italian Obesity Barometer Report", 9 aprile 2019. http://www.ibdo.it/pdf/OBESITY-REPORT-2019. pdf. Accessed on 7 February 2021

47. WHO 2016. https://www.who.int/news-room/fact-sheets/detail/ obesity-and-overweight. Accessed on 7 February 2021

48. Ehehalt F, Saeger HD, Schmidt CM, Grützmann R (2009) Neuroendocrine tumors of the pancreas. Oncologist 14(5):456-467

49. International osteoporosis foundation. https://www.osteoporosis. foundation/facts-statistics/epidemiology-of-osteoporosis-andfragility-fractures. Accessed on 7 February 2021

50. Broken bones, broken lives: a roadmap to solve the fragility fracture crisis in Italy. https://www.osteoporosis.foundation/ sites/iofbonehealth/files/2019-06/4.\%202018_EU6Italy_Report_ BrokenBonesBrokenLives_English.pdf. Accessed on 7 February 2021

51. Natalità e fecondità della popolazione residente. https://www. istat.it/it/files//2019/11/Report_natalit\%C3\%A0_anno2018_def. pdf. Accessed on 7 February 2021

52. Aborti spontanei ed interruzioni volontarie di gravidanze. http:// dati.istat.it/Index.aspx?DataSetCode=DCIS_ABORTISPONTR. Accessed on 7 February 2021

53. Standard italiani per la cura del diabete mellito 2018. https:// www.siditalia.it/pdf/Standard\%20di\%20Cura\%20AMD\%20\%20SID\%202018_protetto.pdf. Accessed on 7 February 2021

54. Linee Guida Nazionali di rieferimento SIGO Tiroide e gravidanza. https://www.sigo.it/wp-content/uploads/2015/10/tiroide_ grav1.pdf. Accessed on 7 February 2021

55. Calina D, Docea AO, Golokhvast KS et al (2019) Management of endocrinopathies in pregnancy: a review of current evidence. Int $\mathrm{J}$ Environ Res Public Health 16(5):781 